\title{
Consistency, precision, and accuracy of optical and electromagnetic shape-capturing systems for digital measurement of residual-limb anthropometrics of persons with transtibial amputation
}

\author{
Mark D. Geil, PhD \\ Department of Kinesiology and Health, Georgia State University, Atlanta, GA
}

\begin{abstract}
Computer-aided design (CAD) and computer-aided manufacturing systems have been adapted for specific use in prosthetics, providing practitioners with a means to digitally capture the shape of a patient's limb, modify the socket model using software, and automatically manufacture either a positive model to be used in the fabrication of a socket or the socket itself. The digital shape captured is a three-dimensional (3-D) model from which standard anthropometric measures can be easily obtained. This study recorded six common anthropometric dimensions from CAD shape files of three foam positive models of the residual limbs of persons with transtibial amputations. Two systems were used to obtain 3-D models of the residual limb, a noncontact optical system and a contact-based electromagnetic field system, and both experienced practitioners and prosthetics students conducted measurements. Measurements were consistent; the mean range (difference of maximum and minimum) across all measurements was $0.96 \mathrm{~cm}$. Both systems provided similar results, and both groups used the systems consistently. Students were slightly more consistent than practitioners but not to a clinically significant degree. Results also compared favorably with traditional measurement, with differences versus hand measurements about $5 \mathrm{~mm}$. These results suggest the routine use of digital shape capture for collection of patient volume information.
\end{abstract}

Key words: amputee, anthropometry, CAD, computer-aided design, digitization, measurement, outcomes, prosthetics, rehabilitation, residual limb.

\section{INTRODUCTION}

The traditional and most widely used technique for manufacturing prosthetic sockets involves the prosthetist first making a negative cast of the residual limb and then filling the cast with plaster to form a positive mold of the residual limb. After the prosthetist makes structural modifications to the positive mold, which generally involve physical carving of the mold through incremental shaving, a socket is made over the plaster model. While such a classical socket manufacturing process is effective under the guidance of skilled prosthetists, problems with this process include variable accuracy and reliability between prosthetists, increased patient time and discomfort, and inaccurate manual structural modifications. These pitfalls may in turn lead to decreased patient satisfaction and elevated costs.

\footnotetext{
Abbreviations: $3-\mathrm{D}=$ three-dimensional, $\mathrm{AP}=$ anteriorposterior, $\mathrm{CAD}=$ computer-aided design, $\mathrm{CAM}=$ computeraided manufacturing, LED = light-emitting diode, ML = medial-lateral, $\mathrm{MPT}=$ midpatellar tendon, $\mathrm{SD}=$ standard deviation, $\mathrm{TD}=$ total difference.

Address all correspondence to Mark D. Geil, PhD; Biomechanics Laboratory, Department of Kinesiology and Health, Georgia State University, PO Box 3975, Atlanta, GA 30302-3975; 404-413-8379; fax: 404-651-4814.

Email: mgeil@gsu.edu

DOI: 10.1682/JRRD.2006.08.0088
} 
The introduction of computer-aided design (CAD) and computer-aided manufacturing (CAM) systems into the prosthetics and orthotics community presented clinicians, technicians, and patients with numerous potential advantages over traditional techniques. Several CAD/ CAM systems are now available and used in prosthetics clinics throughout the United States [1]. These systems differ in their capabilities, but CAD/CAM in the context of socket design generally involves scanning of the residual limb to produce a digital image [2]. Following digitization, the limb model is modified in software and data from the modified limb are sent to an automatic carver that replicates the residual limb, typically using a foam material. The foam model, analogous to the positive plaster model of traditional methods, then serves as a template for socket production [3].

One of the most profound innovations of CAD technology is that the prosthetist can modify the digitized limb in software rather than by sanding, filing, or filling a plaster mold, which can be inaccurate and time-consuming processes, particularly in the hands of unskilled technicians. With the CAD system, however, precise modifications and revisions can be made literally with the click of a computer mouse, and the results of these "virtual" modifications can be observed and measured in software before the final positive mold is actually fabricated. In addition, common modifications, such as indentation at the midpatellar tendon (MPT) site, can be stored and simply applied to digitizations of future patients [4].

In addition to quick and accurate modification of digitized residual limbs, an important advantage of CAD/ CAM is rapid production of the positive mold. The time required to digitize a residual limb has been reported to be several minutes [1], and newer digitizing methodologies allow for scanning in seconds. In addition, fabrication of the sockets from digital data with an automated carver system is rapid (15-30 minutes) and accurate. Decreased time to manufacture means that the total time for the prosthesis fitting process is decreased, which leads to two immediate benefits. First, the medical team requires less time and resources. Second, given that "optimal” limb design varies by patient, being able to produce quick positive molds and easily modify those molds allows the prosthetist to produce several unique biomechanical designs per patient visit [5], increasing the likelihood of providing a sound and comfortable design for the patient.
Other advantages of CAD/CAM in socket manufacture include its ease of integration with central fabrication sites [3], which is particularly relevant to developing countries [6], and its low cost. The inexpensive materials and quick production time suggest that CAD/CAM presents a cost-effective alternative to plaster techniques. More globally, CAD/CAM presents a method for manufacturing inexpensive, reliable sockets in developing countries, as has been demonstrated in the Prosthetics Outreach Foundation's clinic in Hanoi, Vietnam [7]. Finally, for the academic community, structural data obtained from digitization can be stored and used for more sophisticated structural analyses. For example, limb geometry data could be used in computational models of biomechanical forces translated from the residual limb via the socket to other prosthetic limb components during physical activity. Thus, using data from a digitized residual limb to calculate an "ideal" socket design may be possible in the future.

While CAD/CAM techniques offer numerous novel applications and advantages over older techniques, quantitative studies evaluating accuracy, reliability, and costeffectiveness relative to existing methods are lacking. Previous studies have evaluated the precision and reliability of various digital shape-capture systems to make volume measurements of both simple geometric shapes and residual-limb models [8-9]. However, these studies did not evaluate more clinically relevant measurements, such as anterior-posterior (AP) diameter and circumference measurements at the MPT. In addition, the hypothesized enhanced accuracy and reliability of CAD/CAM techniques versus traditional measurement and fabrication techniques have not been directly demonstrated. Inter- and intraprosthetist accuracy and reliability regarding each step of the residual-limb measurement and socket manufacture processes have been quantified for traditional systems, such as tape measures and calipers [10], but not for CAD/CAM methods. Such determinations are important for evaluation of CAD/CAM as a long-term cost-effective strategy for clinical use and may reveal which specific steps of the entire socket manufacturing process can be aided by the newer CAD/CAM technology. Furthermore, establishment of the accuracy, precision, and consistency of digital shape-capture tools in the measurement of standard anthropometrics may demonstrate an underutilized value of digital technologies in clinical practice [11], particularly environments in which the collection of outcome measures is increasingly prevalent [12]. 
This investigation used transtibial unmodified foam residual-limb positive models (1) to evaluate the accuracy and reliability of the digital shape-capture component of two commercially available CAD systems (Tracer OMEGA and T-Ring II; Ohio Willow Wood, Mount Sterling, Ohio) compared with previously recorded and published anthropometrics from standard measurement tools (standard tape measure, force gauge tape measure, anthropometer, VAPC, and Ritz Stick) [10] and (2) to quantify several clinically important residual-limb measurement parameters.

\section{METHODS}

Digital shape capture was conducted for three foam positive models (labeled “A," "B," and "C") of the residual limbs of persons with transtibial amputation. These models were identical to those used in Geil [10], which enabled comparison of digitized data with traditional caliper and tape measure data. Each model was mounted on a polyvinylchloride shaft and covered with two Cool Blue (LTWT lightweight $6 \times 3 \times 12$ in.) prosthetic socks (SPS by Knit-Rite, Alpharetta, Georgia) and a white $6 \mathrm{~mm}$ Alpha Uniform C-Liner (Ohio Willow Wood, Mount Sterling, Ohio) suitable for use with the T-Ring II (Figure 1). The socks and liner allowed for some compression, mimicking soft tissue. Unbeknownst to the study participants, models $\mathrm{A}$ and $\mathrm{C}$ were identical, which enabled withinsubject repeatability assessment. Because this study assessed instrument accuracy and consistency and instrument use, as opposed to anatomical knowledge and palpation technique, anatomical landmarks were identified with small black dots (identifiable by the T-Ring II) on the Alpha liner covering each model. The following locations were identified: MPT, medial and lateral marks at the midpatella line, 2 in. distal to the MPT on the anterior aspect, and $4 \mathrm{in}$. distal to the MPT on the anterior aspect. The MPT mark was used for the AP, length, and circumference measurements; the medial and lateral marks were used for the medial-lateral (ML) measurement; and the marks 2 and $4 \mathrm{in}$. distal to the MPT were used for additional circumference measurements.

Each model was secured in a vise, and participants were asked to digitize the shape of each model using the digital shape-capture devices from two different CAD systems: the Tracer OMEGA system and the T-Ring II system, which are both manufactured by Ohio Willow Wood (Mount Sterling, Ohio) and operate off the same software platform (TracerCAD Premier v. 8.0.2; Ohio Willow Wood, Mount Sterling, Ohio).

The Tracer OMEGA system is a contact scanner that identifies the three-dimensional (3-D) position and orientation of a small "pen" in a magnetic field. The operator defines the shape of the model by placing the pen in contact with the model and tracing the entire surface area. The resulting shape of the model is therefore sensitive to the pressure applied by the operator while tracing. The T-Ring II system is a noncontact optical scanner. A ring containing four optical cameras and four light-emitting diode (LED) arrays is held perpendicular to the long axis of the limb. The LED arrays project parallel lines onto a white liner covering the limb. Changes in spacing between the lines that correspond to out-of-plane contours of the limb shape are recorded by the cameras and the 3-D shape is reconstructed. The software automatically detects the black dots placed over the landmarks and identifies these locations on the digital model (Figure 1). Data capture occurs in less than 1 second and involves no contact with the overall surface of the limb. Consequently, the T-Ring II system is not sensitive to operator scanning technique but is sensitive to the orientation at which the device is held with respect to the limb.

Participants were recruited from two populations: practitioners and students. Practitioners were certified in prosthetics or orthotics by the American Board for Certification in Orthotics, Prosthetics \& Pedorthics and had completed the Ohio Willow Wood training course for and had experience using the two CAD systems. Students were first- or second-year students in the Georgia Institute of Technology Master of Science program in Prosthetics and Orthotics; all had completed a semester-long course in CAD/CAM that included the Ohio Willow Wood training course for both CAD systems in this study but had no practical or clinical experience using the systems.

Four practitioners and seven students completed the study. Each provided informed consent prior to participation, and the Georgia State Institutional Review Board approved the study. Digitization type and model order were randomized for each participant. Each participant was given specific instructions for each digital shapecapture system. For the contact scanner, participants were instructed to trace the model, use the pen for large blends following tracing, and identify the aforementioned landmarks following a custom Tracer sequence. No additional modifications were permitted. For the optical scanner, participants were permitted to use either of two 


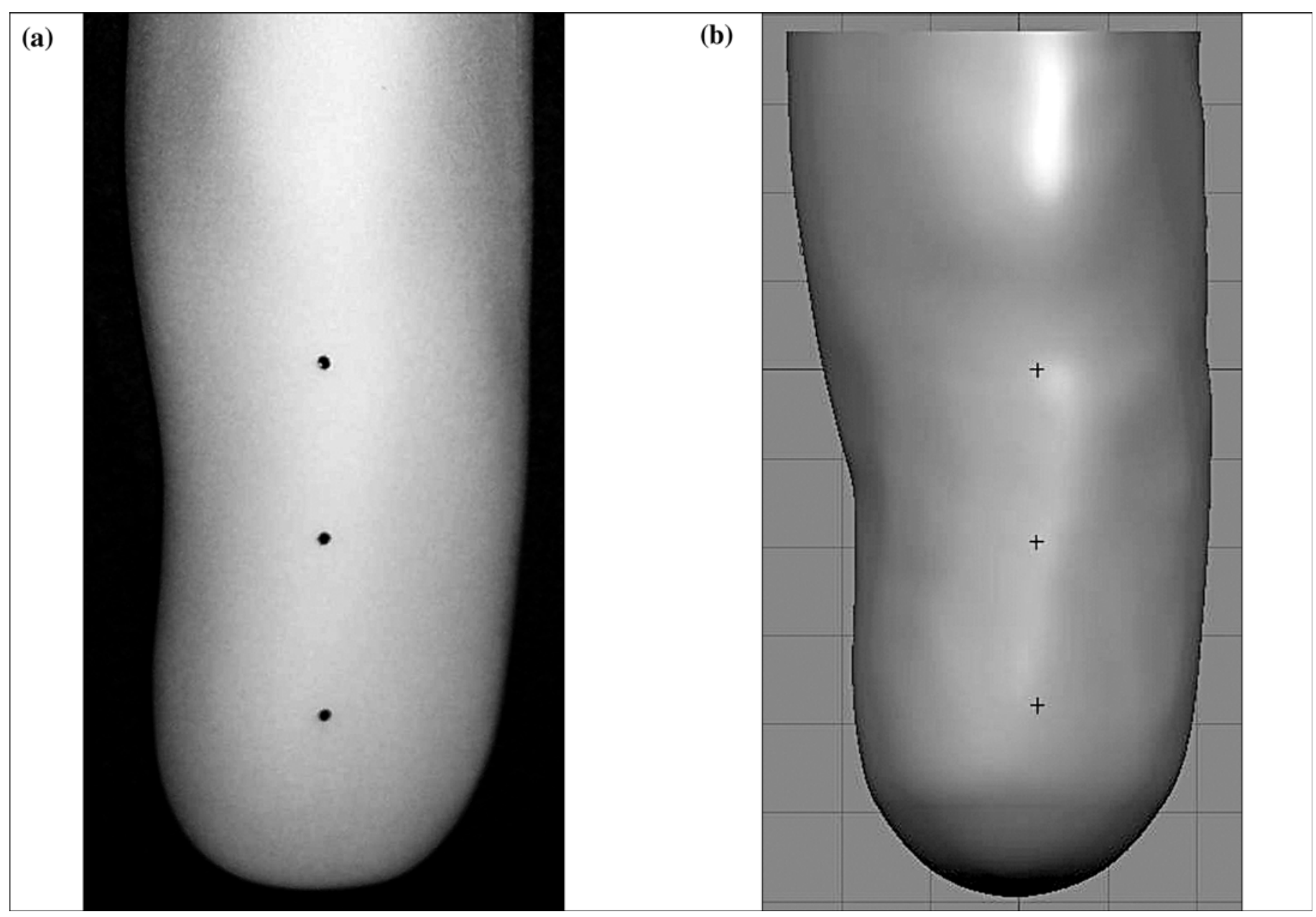

Figure 1.

Residual-limb model A: (a) photograph of foam positive model, with landmarks indicated by dots and (b) TracerCAD Premier software model (Ohio Willow Wood, Mount Sterling, Ohio), with landmarks indicated by crosshairs and longitudinal and medial-lateral axes displayed.

possible rotational orientations of the ring based on personal preference. Participants captured the image and were allowed to use the software for basic orientation corrections. No blends or subsequent modifications were permitted. In approximately 15 percent of cases, scans were repeated when not all landmarks were identified by the software, typically because of insufficient ambient light conditions. Repeat scans add only a few seconds to the total capture time.

The 11 participants capturing two digital images of each of three limb models produced a total of 66 model files. Each model file was labeled with the limb model label and the participant code. Following data collection, anthropometric measurements were recorded at the identified landmarks for each of the model files. The software automatically identifies the measurements as follows. A baseline cylindrical model is rotationally aligned in 3-D space by prompting the user to indicate anterior, medial, and lateral locations. The length of the limb is determined by prompting the user for distal and proximal locations, and the distal location is used to direct the long axis of the limb. AP diameter is the linear distance perpendicular to the long axis of the limb and oriented along the AP axis. ML diameter is also perpendicular to the long axis and oriented along the ML axis. Circumference is the distance around the surface contour at a given level perpendicular to the long axis of the limb. In some cases, the medial and lateral marks at the MPT were not coplanar in the limb's transverse plane and were therefore not in the same transverse slice of the limb model. If the 
circumferences at each landmark were consequently unequal, the average of the two circumferences was recorded. In no case did the difference between the two circumferences exceed $4.0 \mathrm{~mm}$ (mean \pm standard deviation [SD] difference $0.18 \pm 0.08 \mathrm{~mm}$ ).

Linear AP distance and length were recorded at the level of the MPT landmark. ML distances were recorded at the medial and lateral marks. Circumferences were recorded at the level of the MPT and at 2 and 4 in. distal.

Data were analyzed to address several specific questions:

- How precise and consistent were measurements by different participants at a given site?

- Which group (students vs practitioners) was more consistent?

- Did the two systems (optical vs contact) produce similarly accurate and precise results?

- How did the accuracy of results of digitally captured shape measurement compare with "gold standard" analog data?

An overall measure of consistency at each measurement site was obtained by assessment of the range of results (maximum minus minimum) and the SD at each measurement site across all participants and systems. The analysis assessed the general usefulness of anthropometric data as obtained by digital capture systems in the context of clinical significance. After an exploratory statistics module (SPSS 11.0.1, Chicago, Illinois) generated mean and SD values and screened the data for outliers, data were compared by subject group (students vs practitioners). In addition, each measurer's error in the repeated measurements of identical models $\mathrm{A}$ and $\mathrm{C}$ was calculated as the absolute value of the difference between $\mathrm{A}$ and $\mathrm{C}$ measurements. The mean error and maximum error were determined for each participant. A similar comparison was made between systems and across participants. Consistency between the two digital capture systems was calculated as the absolute value of the difference in each participant's measurement using the contact system model and the optical system model.

These absolute values were added for each measurement location $(m)$, providing a total difference (TD) (optical vs contact) for each participant (Equation):

$$
\mathrm{TD}=\sum_{m=1}^{m=6}\left|m_{\text {contact }}-m_{\text {optical }}\right| .
$$

Finally, results were directly compared to previously published data from a study that used analog hand tools
[10]. For assessment of accuracy, the results were compared with previous data on the same models and landmarks. These data identified accurate linear measurements using a GPM anthropometer (SiberHegner, Zurich, Switzerland), which is often used to record body segment parameters in a gait analysis laboratory, and circumference measurements Spring Tape (Tech-Med model 4414, Tech-Med Services, Inc; Hauppauge, New York). This flexible tape measure incorporates a spring on the end with a mark identifying a standard amount of tension.

\section{RESULTS}

Prior to analysis of groups and tools, measurement results were averaged across all participants and both digital capture systems. The mean, SD, and range were recorded for each model and measurement (Table). All measurement sites produced consistent measurements. The largest range recorded across the 11 participants and two measurement systems was $1.70 \mathrm{~cm}$, at the length measurement. This maximum range was the difference between the Model C lengths (MPT to distal end) of $20.01 \mathrm{~cm}$ and $18.40 \mathrm{~cm}$ measured by different practitioners both using the contact scanner. The mean range across all measurements was $0.96 \mathrm{~cm}$.

Group consistency was assessed, first, by a comparison of the SD and range values and, second, by a comparison of each group's ability to consistently measure identical models A and C. Across all models, students were more consistent than practitioners, as assessed by both range (students showed a smaller range in $67 \%$ of measurements) and SD (students showed a smaller SD in $56 \%$ of measurements). Differences were slight, however. For example, the sum of student ranges across all measurements and all models was $13.00 \mathrm{~cm}$ compared with $13.35 \mathrm{~cm}$ for practitioners. Comparison of model A versus C error revealed sources of inconsistency (Figure 2). Practitioners showed a larger average error in the length measurement than did the students, while other sites showed very similar errors. In the model A versus C comparison, students' models showed more consistency in all linear measures, while practitioners' models showed more consistency in all circumferential measures.

Two digital shape-capture systems were used in the study: an optical system (T-Ring II) and a contact scanner (Tracer OMEGA). Just as group results were consistent, results between systems, measured as the TD (optical vs contact) for each participant (Equation), were also 
Table.

Mean \pm standard deviation (SD) and range (difference of maximum and minimum) for three positive foam models (A, B, and C) and different measurement locations across systems and participant populations. All data shown in centimeters.

\begin{tabular}{|c|c|c|c|}
\hline $\begin{array}{c}\text { Measurement } \\
\text { Location }\end{array}$ & A & B & C \\
\hline \multicolumn{4}{|l|}{$\overline{\mathbf{A P}}$} \\
\hline Mean \pm SD & $13.41 \pm 0.45$ & $10.97 \pm 0.15$ & $13.40 \pm 0.19$ \\
\hline Range & 1.40 & 0.50 & 0.70 \\
\hline \multicolumn{4}{|l|}{ Length } \\
\hline Mean \pm SD & $18.90 \pm 0.36$ & $14.60 \pm 0.37$ & $19.09 \pm 0.40$ \\
\hline Range & 1.50 & 1.40 & 1.70 \\
\hline \multicolumn{4}{|l|}{ ML } \\
\hline Mean \pm SD & $13.28 \pm 0.17$ & $11.62 \pm 0.11$ & $13.29 \pm 0.12$ \\
\hline Range & 0.70 & 0.45 & 0.40 \\
\hline \multicolumn{4}{|l|}{ Circ MPT } \\
\hline Mean \pm SD & $40.51 \pm 0.17$ & $33.90 \pm 0.22$ & $41.15 \pm 0.25$ \\
\hline Range & 0.70 & 0.70 & 0.80 \\
\hline \multicolumn{4}{|l|}{ Circ +2} \\
\hline Mean \pm SD & $40.02 \pm 0.22$ & $32.34 \pm 0.22$ & $40.66 \pm 0.26$ \\
\hline Range & 0.90 & 1.00 & 1.00 \\
\hline \multicolumn{4}{|l|}{ Circ +4} \\
\hline Mean \pm SD & $39.19 \pm 0.25$ & $31.00 \pm 0.31$ & $39.92 \pm 0.33$ \\
\hline Range & 1.00 & 1.20 & 1.20 \\
\hline
\end{tabular}

$\mathrm{AP}=$ anterior-posterior distance at midpatellar tendon, Length $=$ linear distance from midpatellar tendon (MPT) to distal end, ML = average of mediallateral distances at medial and lateral landmarks at MPT level; Circ MPT $=$ circumference (circ) at level of MPT, Circ $+2=$ circ 2 in. distal to MPT, Circ $+4=$ circ 4 in. distal to MPT.

consistent. The single largest overall TD was $3.75 \mathrm{~cm}$, representing an average error of $6.2 \mathrm{~mm}$ when divided by the six measures. The largest actual system difference for a single measure was $1.50 \mathrm{~cm}$, when a practitioner recorded a length of $18.50 \mathrm{~cm}$ for model $\mathrm{C}$ using the optical system and $20.00 \mathrm{~cm}$ using the contact system.

Model A versus C error was also used to assess system differences. Both systems were largely accurate in replicating similar measurements for the identical models (Figure 3). Neither system consistently overestimated or underestimated the linear differences, though errors were greatest for circumferential measures taken from optically captured models. Despite this trend, none of the errors observed could be considered clinically significant, especially because the entire range of the $y$-axis in Figure 3 is $1 \mathrm{~cm}$.

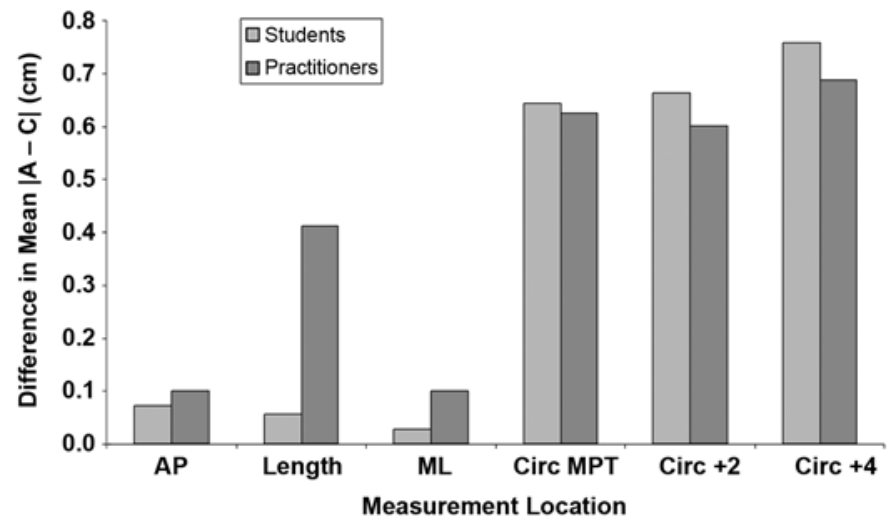

Figure 2.

Measurement error between identical models A and C by location and across both digital shape-capture systems. Shown as absolute value of mean distance or circumference (circ) on model A minus mean distance or circ on model $\mathrm{C}$ for all students and practitioners at each measurement location. $\mathrm{AP}=$ anterior-posterior, $\mathrm{Circ}+2=$ circ 2 in. distal to midpatellar tendon (MPT), Circ $+4=$ circ 4 in. distal to MPT, Length $=$ linear distance from MPT to distal end, ML = medial-lateral.

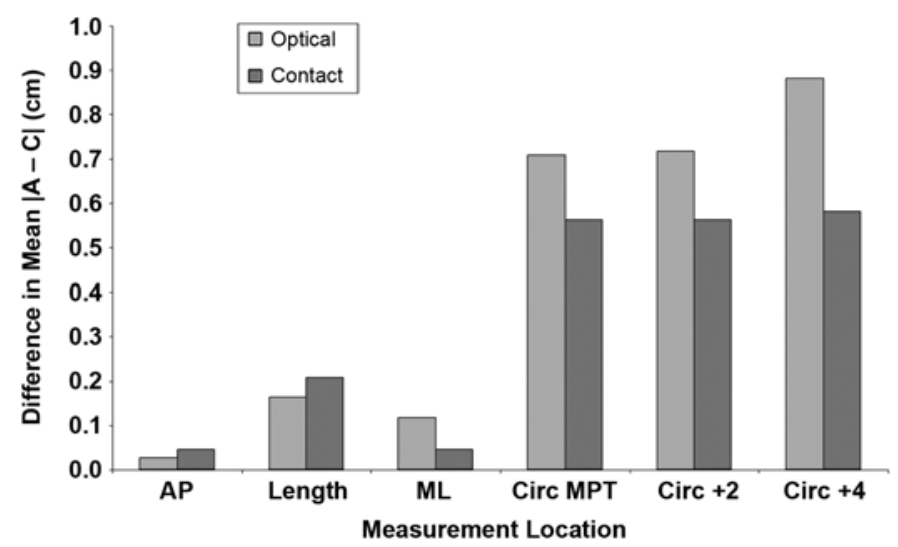

Figure 3.

Measurement error between identical models A and C by location and across participants. Shown as absolute value of mean distance or circumference (circ) on model A minus mean distance or circ on model C for each measurement system (T-Ring II scanner [Optical] vs Tracer OMEGA magnetic field-based scanner [Contact], both by Ohio Willow Wood, Mount Sterling, Ohio) at each measurement location. AP = anterior-posterior, Circ +2 = circ 2 in. distal to midpatellar tendon (MPT), Circ $+4=$ circ 4 in. distal to MPT, Length $=$ linear distance from MPT to distal end, $\mathrm{ML}=$ medial-lateral.

A final analysis compared the digital results to previously published anthropometrics collected with hand tools [10]. Again, results were quite similar. When results for each measurement from each study were averaged across all subjects (Figure 4), differences between average data from the present study and average data using the gold standard hand tools (anthropometer and spring-loaded 
(a)

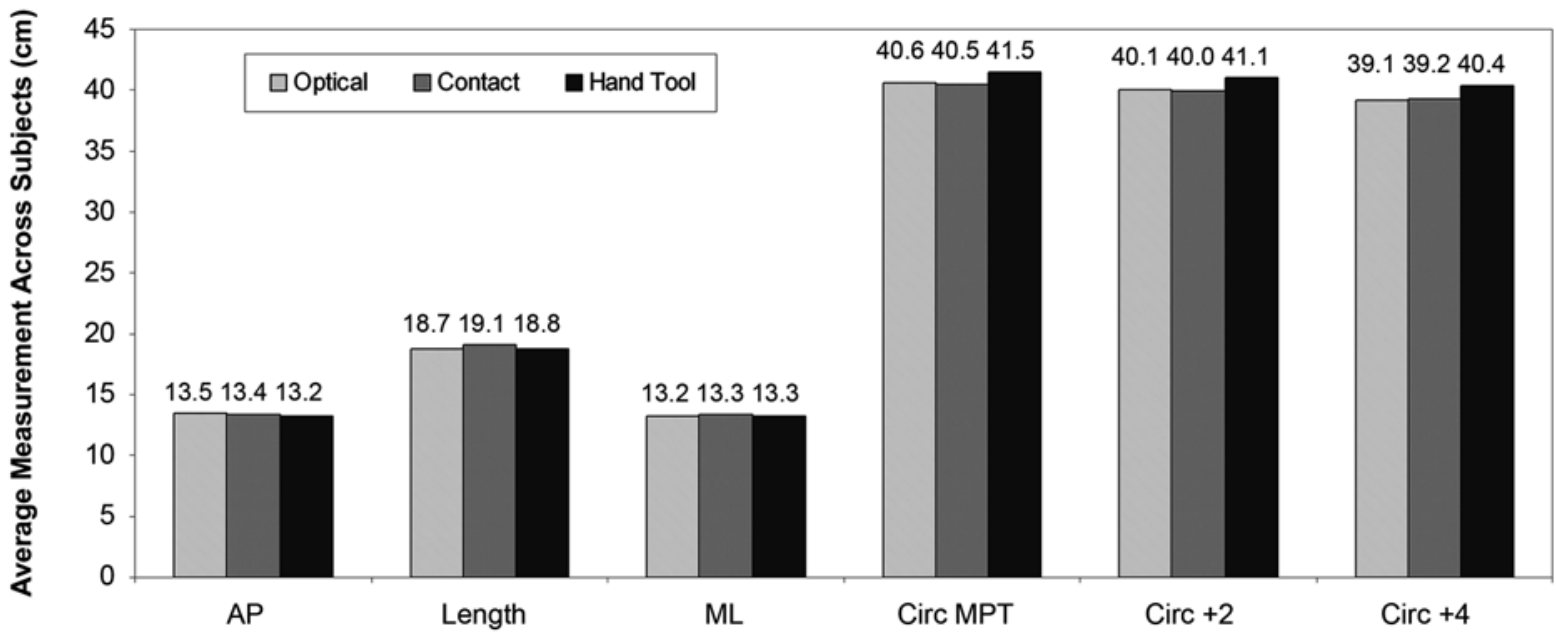

(b)

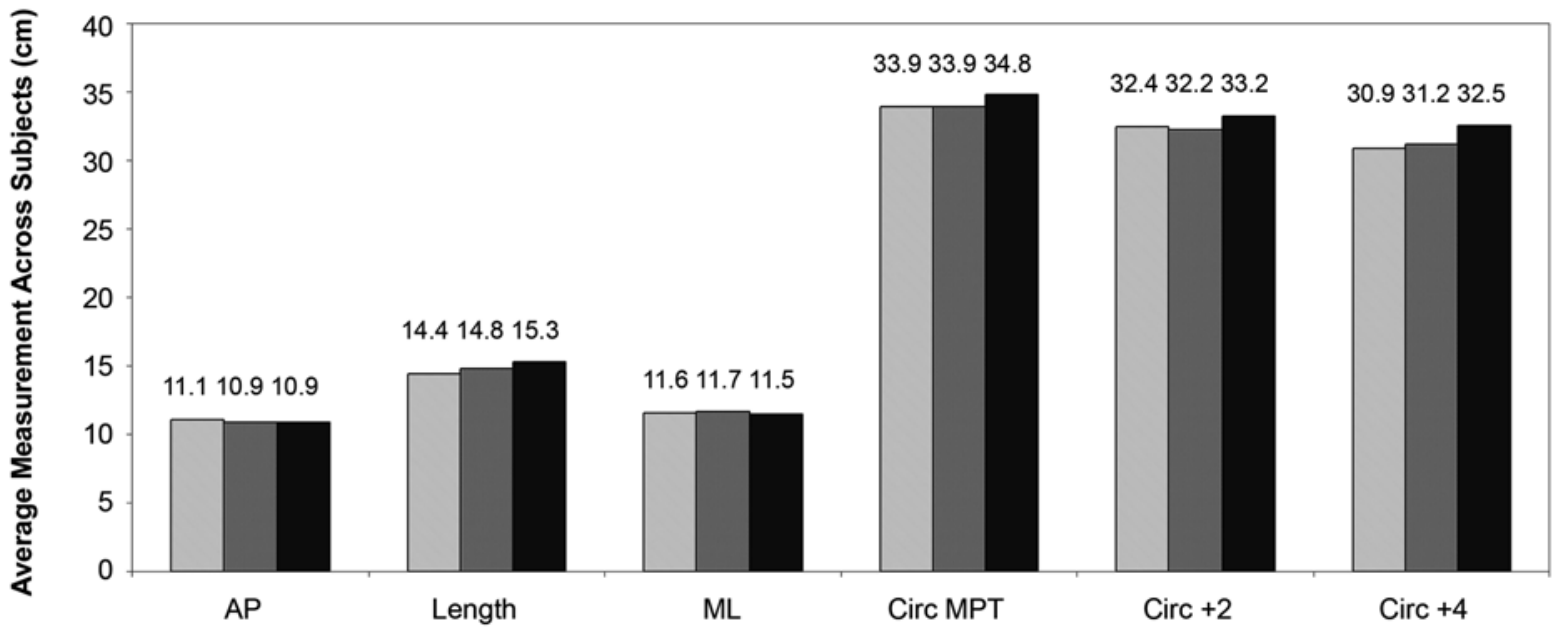

(c)

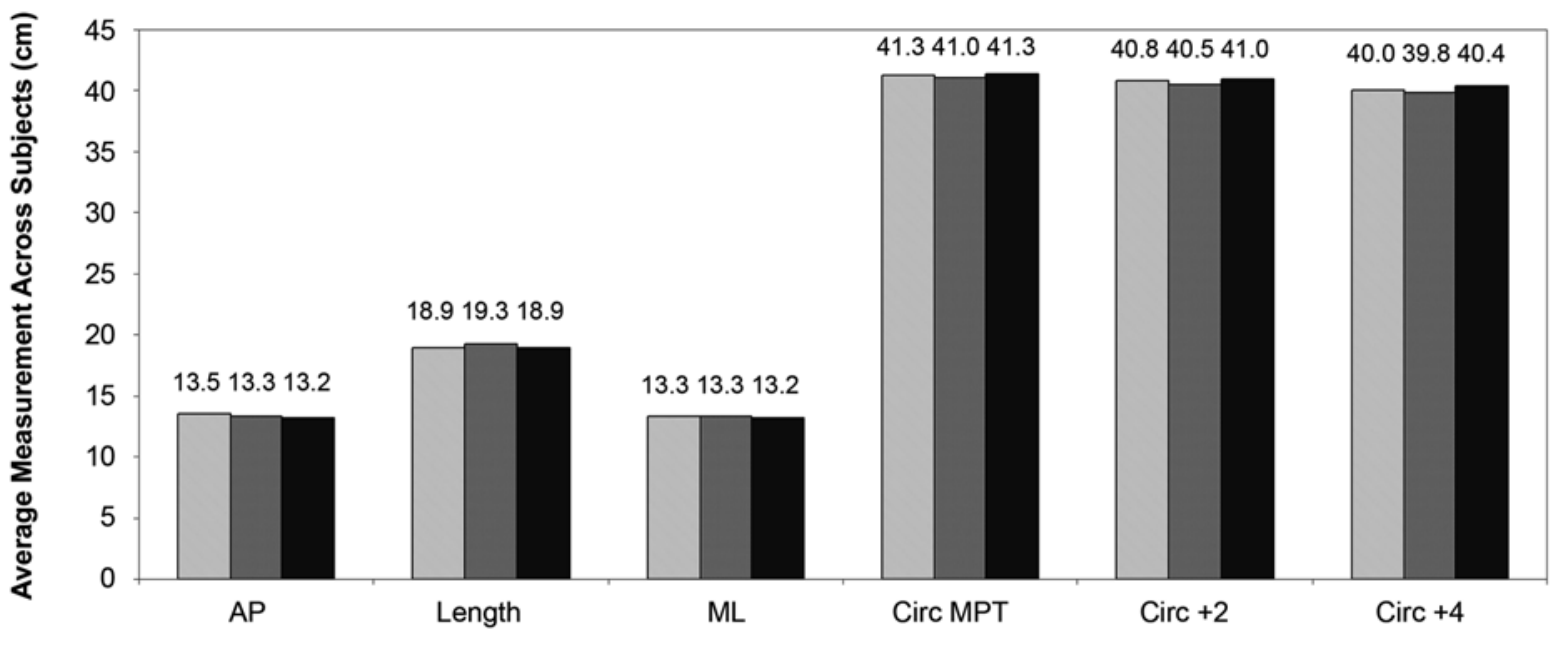

Figure 4.

Average measurement by location across all students and practitioners in current study using T-Ring II scanner (Optical) and Tracer OMEGA magnetic field-based scanner (Contact) vs multiple students and practitioners measuring same three models using standard calipers or tape measures (Hand Tool). Data presented for (a) model A, (b) model B, and (c) model C (identical to A). AP = anterior-posterior, Circ $=$ circumference, Circ $+2=$ circ 2 in. distal to midpatellar tendon (MPT), Circ $+4=$ circ 4 in. distal to MPT, Length = linear distance from MPT to distal end, ML = medial-lateral. 
tape measure) were approximately $0.5 \mathrm{~cm}$. The largest differences between digital and hand tools were found in circumferential measures.

\section{DISCUSSION}

This study investigated the use of digital shapecapture methods to record standard anthropometrics of models of the residual limbs of persons with transtibial amputation. The study compared the consistency of two CAD systems and their use by two populations of measurers. If the systems can be established as accurate and consistent, and the results they produce compare favorably with similar measurements with conventional hand tools, practitioners may wish to consider routinely using digital shape-capture systems for anthropometric measurement.

The study was limited in its scope. Only two digital shape-capture systems were considered. The study did not test the full capability of CAD systems to capture and also modify a model. Some participants in the study noted that, in practice, they would normally modify their models substantially more than was allowed in this study. An additional limitation was that mean and SD values were affected by the difference in sample size for each group (seven vs four). This limitation would have been of greater concern had larger differences been noted in any of the measures. Finally, the study should be recognized as a cross-section of measurements that does not consider the consistency of measurements done by the same person over a span of months or years, a scenario with particular clinical relevance.

Measurement results were consistent across systems and participant groups. An ensemble average measurement range of $<1 \mathrm{~cm}(0.96 \mathrm{~cm})$ is clinically acceptable, particularly considering that the measure is across two different digital shape-capture systems and two very different participant populations. Differences were present between groups, but no result suggested clinically significant differences in consistency or measurement error between the students and practitioners. This result implies that a baseline level of training is all that is required to produce consistent and accurate anthropometric measurements with digital shape capture. This result does not imply that experience does not factor in the proper use of CAD/CAM systems for the design and fabrication of a prosthetic socket. The present experiment was designed to assess the utility of CAD systems in the collection of anthropometric mod- els; consequently, modifications (which are essential to the socket design process) were excluded from the protocol. Clinical experience almost certainly plays a larger role in model modification than in proper digital shape capture.

Some technological differences could explain why length was the most inconsistently measured location. CAD systems start with a uniform model upon which scanner data either add or delete volume in slices. A challenging scenario for shape digitization occurs when the model requires a closed end in a plane perpendicular to the long axis of the cylinder. Such is the case with residual-limb models and prosthetic sockets, suggesting that end effects may produce less consistent results for length measures not because of the location of the MPT landmark but rather the location of the distal end along the model's long axis.

Though the systems used in this study share the same modeling software, their physical and mechanical uses are quite different and help explain the model $A$ versus $C$ error between systems (Figure 3). The fact that the optical scanner must be held perpendicular to the long axis of the residual limb implies that errors may occur if that angle varies. The results of this study revealed that circumferential measurements were more variable with the optical system, suggesting that these measures may be more sensitive to errors in perpendicularity. Figure 5 demonstrates that a relatively small $5^{\circ}$ error produces a "perpendicular" diameter that is longer than the true diameter. Linear distances are subject to this error only if the tilt occurs in the same plane, but circumferences are affected by any radial tilt. The $5^{\circ}$ tilt shown in Figure 5 produces only 0.76 percent error in diameter (twice the inverse of the cosine of $5^{\circ}$ ), but larger tilt angles produce larger errors. Practitioners using optical scanners for circumferential measurements should pay particular attention to the perpendicularity of the scanner when measuring.

Digitally captured data were surprisingly similar to measurements with conventional hand tools. This result suggests that, at least for linear and circumferential shape measures at distinct landmarks, measures obtained by either digital system in comparison with analog measurement with hand tools have no clinically significant differences. Slightly larger differences did occur between the digital systems and the hand tools in the circumference measurements, which is somewhat surprising. One would expect the tension developed in a tape measure to reduce results compared with the surface circumference recorded by the digital systems. However, the hand-measured circumferences were consistently larger (Figure 4). 


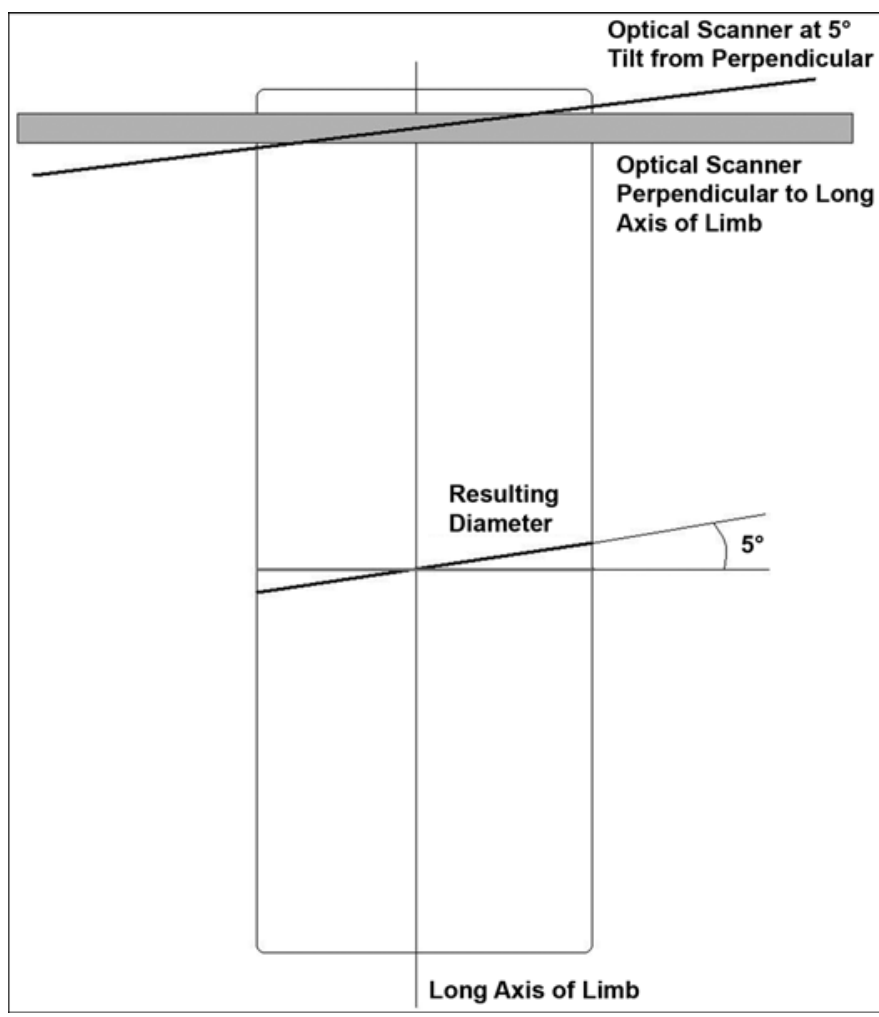

Figure 5.

Effect of optical scanner tilt on diameter and therefore circumference. Tilt of $5^{\circ}$ from proper position (perpendicular to long axis of limb) causes overestimation of diameter (by $0.76 \%$ ).

One should note that digital systems are used differently than conventional tools. In this case, the digital systems collected far more information more efficiently. The optical capture system used in this study records data in less than 1 second. Most participants completed the contact scanner data collection in 1.5 to 3.0 minutes per scan. A brief amount of time is associated with labeling landmarks and obtaining the anthropometrics, but this step requires only a few mouse clicks. Hand measurement of anthropometrics takes as long or longer than either digital capture system, and the digital systems provide far more data since the complete 3-D model is recorded, not just the six anthropometric measures.

Most practitioners assume that CAD/CAM systems in prosthetics are to be used only for designing and fabricating a prosthetic socket. The study results suggest an additional use for the digital shape-capture component of the systems. Recording data related to the shape of each patient's residual limb, including standard anthropometric measures, can be accomplished accurately, consistently, and efficiently with digital shape capture, even if the socket is subsequently produced by conventional means.

\section{CONCLUSIONS}

Anthropometric measurements taken from digitally captured models were accurate and consistent. The optical and contact scanners tested produced similar results for the same measurement, and both students and practitioners were able to use the devices consistently. Results were also similar to those obtained from hand calipers and tape measures. Digital shape capture may be efficiently used to record residual limb anthropometrics and 3-D shape information.

\section{ACKNOWLEDGMENTS}

I extend thanks to the participants in the study and to Matthew Tate, PhD, Emory University, for assistance in preparation of the manuscript.

This material is the result of work supported with resources and the use of facilities at the Department of Kinesiology and Health, Georgia State University, Atlanta, Georgia.

The author has declared that no competing interests exist.

\section{REFERENCES}

1. Houston VL, Burgess EM, Childress DS, Lehneis HR, Mason CP, Garbarini MA, LaBlanc KP, Boone DA, Chan $\mathrm{RB}$, Harlan $\mathrm{JH}$, Brncick MD. Automated fabrication of mobility aids (AFMA): Below-knee CAD/CAM testing and evaluation program results. J Rehabil Res Dev. 1992; 29(4):78-124. [PMID: 1432729]

2. Boone DA, Burgess EM. Automated fabrication of mobility aids: Clinical demonstration of the UCL computer-aided socket design system. J Prosthet Orthot. 1989;1(3):187-90.

3. Smith DG, Burgess EM. The use of CAD/CAM technology in prosthetics and orthotics - Current clinical models and a view to the future. J Rehabil Res Dev. 2001;38(3):327-34. [PMID: 11440264]

4. Boone DA, Harlan JS, Burgess EM. Automated fabrication of mobility aids: Review of the AFMA process and VA/ Seattle ShapeMaker software design. J Rehabil Res Dev. 1994;31(1):42-49. [PMID: 8035359$]$

5. Murdoch G. Editorial. Prosthet Orthot Int. 1985;9(1):1-2. 
6. Boone DA, Urban ND, Smith DG, Burgess EM, Mathews $\mathrm{DE}$, Coleman KL. Use of CAD/CAM for prosthetic services in the developing world. Proceedings of the 9th World Congress of the International Society for Prosthetics and Orthotics; 1998 Jun 30-Jul 3; Amsterdam, the Netherlands. Copenhagen (Denmark): ISPO; 1998. p. 74-76.

7. Smith DG, Boone DA, Harlan JS, Forsgren SM, Burgess EM. Automated prosthetics fabrication in the developing world: The experimental prosthetics center in Hanoi, Vietnam. Orthopaedic J. 1993;2:49-60.

8. Lilja M, Oberg T. Volumetric determinations with CAD/ CAM in prosthetics and orthotics: Errors of measurement. J Rehabil Res Dev. 1995;32(2):141-48. [PMID: 7562654]

9. Johansson S, Oberg T. Accuracy and precision of volumetric determinations using two commercial CAD systems for prosthetics: A technical note. J Rehabil Res Dev. 1998; 35(1):27-33. [PMID: 9505250]

10. Geil MD. Consistency and accuracy of measurement of lower-limb amputee anthropometrics. J Rehabil Res Dev. 2005;42(2):131-40. [PMID: 15944877]

11. Lunsford TR. Clinical research. J Prosthet Orthot. 1993; 5(4):101-4.

12. Convery P, Buis AW, Wilkie R, Sockalingam S, Blair A, McHugh B. Measurement of the consistency of patellartendon-bearing cast rectification. Prosthet Orthot Int. 2003; 27(3):207-13. [PMID: 14727701]

Submitted for publication August 8, 2006. Accepted in revised form March 29, 2007. 